\title{
Caracterización morfológica y faneróptica de hembras Ovino de Pelo Criollo Colombiano "OPC" Sudán
}

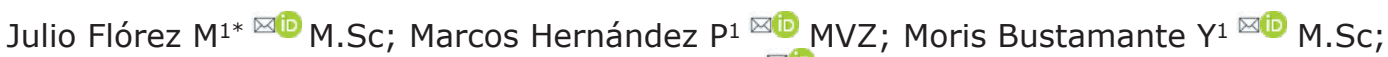 \\ Oscar Vergara $\mathrm{G}^{1} \bowtie \mathbb{D}$ Ph.D.
}

\begin{abstract}
${ }^{1}$ Universidad de Córdoba, Facultad de Medicina Veterinaria y Zootecnia, Departamento de Ciencias Pecuarias, Grupo de Investigación en Producción Animal Tropical (GIPAT), Montería, Colombia.

*Correspondencia: juliomateo7@hotmail.com
\end{abstract}

Recibido: Febrero 2019; Aceptado: Agosto 2019; Publicado: Enero 2020.

\section{RESUMEN}

Objetivo. Esta investigación tuvo como objetivo describir la morfología cualitativa y faneróptica de hembras Ovino de Pelo Criollo Colombiano (OPC) Sudán. Materiales y métodos. Se evaluaron seis características fanerópticas y siete morfológicas cualitativas en 115 hembras Sudán Bayo y Sudán Blanco, de los departamentos Córdoba, Cesar y La Guajira. Los datos obtenidos se analizaron a través del programa estadístico InfoStat ${ }^{\circledR}$ y se estimaron las frecuencias relativas y absolutas para cada uno de los caracteres evaluados. Resultados. Los OPC Sudán Bayo se distinguieron por ser de un color de capa bayo amarillo. Los Sudán Blanco fueron de capa overo castaño, pero predominando siempre el blanco sobre el castaño. Estos ovinos se caracterizaron por poseer mucosas negra-rosadas y en menor proporción habían individuos con mucosas rosadas. Además, presentaron orejas horizontales siempre, perfiles subconvexos en más del $80 \%$ de los casos, cuellos generalmente medianos y poco musculados, grupas usualmente inclinadas, ubres parcialmente pigmentadas y también despigmentadas. Asimismo, se caracterizaron por ostentar pezuñas veteadas mayoritariamente, aunque con un alto porcentaje de pezuñas claras en las Sudán Blanco. Conclusiones. Este trabajo ha permitido caracterizar de manera específica a las OPC Sudán desde la morfología y la faneróptica, obteniéndose así información de importancia para delimitar los parámetros de pertenencia a este grupo y para la propuesta de creación de un estándar racial.

Palabras clave: Fenotipo, Oveja, Razas nativas, Recursos genéticos (Fuentes: National agricultural library, AGROVOC thesaurus).

\section{ABSTRACT}

Objective. The objective of this research was to describe the qualitative morphology and phaneroptic of Sudán Colombian creole woolless sheep (OPC). Materials and methods. Six phaneroptic and seven qualitative morphological characteristics were evaluated in a total of 115 Sudán Bayo and Sudán Blanco ewes, from the departments Córdoba, Cesar and La Guajira. The data obtained were analyzed through the statistical program InfoStat ${ }^{\circledR}$, relative and absolute frequencies were estimated for each evaluated trait. Results. Sudán Bayo OPC were distinguished by being yellow coat color. Sudán Blanco were white-and-chestnut spotted coat color, but white always predominated over

Como citar (Vancouver).

Flórez MJ, Hernández PM, Bustamante YM, Vergara GO. Caracterización morfológica y faneróptica de hembras Ovino de Pelo Criollo Colombiano "OPC" Sudán. Rev MVZ Cordoba. 2020; 25(1):e1263. DOI: https://doi.org/10.21897/rmvz.1263 
chestnut. These ovines usually had black-rosy mucosae and in lesser amount there were individuals with rosy mucosae. Besides, they presented horizontal ears always, sub-convex profile in more than $80 \%$ of cases, generally medium-sized and scant muscled necks, usually inclined rumps, partially pigmented and depigmented udders as well. Likewise, they were characterized by showing marbled hooves mostly, but with a high percentage of clear hooves in Sudán Blanco sheep. Conclusions. This research has allowed to characterize specifically. Sudán OPC sheep from morphology and phaneroptic; thus, important information has been obtained to delimit the parameters of belonging to this group and for proposing the creation of a breed standard.

Keywords: Phenotype, ewe, landraces, animal genetic resources (Sources: National agricultural library, AGROVOC thesaurus).

\section{INTRODUCCIÓN}

La llegada al continente Americano de los ovinos de pelo se remonta al segundo viaje hecho por Cristóbal Colón (1), y no se descarta la llegada de animales en barcos dedicados a la esclavitud (2), pero se hace hincapié en que para cuando se realizó el traslado de esclavos africanos al nuevo mundo, ya las islas del Caribe contaban con muchos ovinos de pelo provenientes de las islas Canarias. Ello permitió que se diera la difusión de las razas ovinas de pelo por toda América continental desde las bases caribeñas, lo cual deja entrever que no es coincidencia la gran semejanza fenotípica que tiene la raza ovina de pelo Canaria, de España, con ovinos de pelo criollos americanos como los Pelibuey (3) o los Sudán.

Los Ovinos de Pelo Criollos Colombianos (OPC) Sudán y Etiope han sido resultado de los eventos narrados en el párrafo anterior, y aunque a nivel nacional no se ha unificado un criterio en relación a considerarlos como una o dos razas, se ha sugerido desde la genética por Vivas (4) y desde lo morfoestructural por Flórez et al (5), que los Sudán y Etiope se deben catalogar racialmente diferentes.

Los OPC Sudán son un recurso valioso para el productor del campo colombiano, ya que a pesar de que los sistemas de manejo empleados para su cría, en su gran mayoría han sido extensivos y poco tecnificados, donde se requiere mejorar especialmente los sistemas de alimentación para lograr una mayor tasa de crecimiento, han podido mantenerse en las condiciones del trópico bajo colombiano, demostrando adaptación y elevada prolificidad $(4,6)$. Esto los convierte en una alternativa para contribuir al desarrollo de las comunidades, en especial de las menos favorecidas, que son las principales conocedoras y poseedoras de estos ovinos (7).

En los últimos años los Sudán se han encontrado amenazados a raíz de la introducción de razas exóticas que supuestamente poseen una mayor productividad, y se ignora la rusticidad de los animales criollos (8). Esta amenaza se fundamenta en que una raza no sólo puede estar en peligro desde el punto de vista de la reducción numérica de sus ejemplares, sino también por procesos de hibridación, que en el caso de esta población han sido muy acusados (5). El panorama de esta situación empeora, cuando se tiene en cuenta la carencia de información censal de los OPC.

En estas condiciones, es clara la necesidad de preservar la integridad genética de los OPC Sudán para aprovechar sus cualidades, tanto productivas como de rusticidad en cruzamientos comerciales. Para ello, es necesario que se haga inicialmente una caracterización morfológica rigurosa de esta población, ya que no existen criterios de delimitación racial definidos, e inclusive, no es clara ni se ha concretado aún su condición como raza o subpoblación que pertenece a una raza, siendo todo esto de importancia para poder conservar y fomentar a los Sudán.

Con el fin de contribuir a la definición y consenso sobre lo concerniente al estado racial de los OPC Sudán se ha realizado esta investigación, en la cual se llevó a cabo una descripción morfológica que consistió en evaluar aspectos del exterior animal, dentro de los cuales se consideran los caracteres cualitativos, cuya apreciación es subjetiva, y las variables fanerópticas, término que hace alusión de manera puntual a los caracteres de la piel que se pueden apreciar a simple vista y a producciones dérmicas (9).

Por lo tanto, este trabajo tuvo como objetivo describir de manera detallada los patrones morfológicos cualitativos y fanerópticos de las Ovejas de Pelo Criollo Colombiano Sudán, con el propósito de proporcionar bases que delimiten la clase de ejemplares que pueden pertenecer a 
esta entidad étnica, lo que a futuro contribuirá a establecer un estándar racial y direccionar correctamente planes de mejora genética.

\section{MATERIALES Y MÉTODOS}

Tipo de estudio. Se realizó un estudio descriptivo, de corte transversal. El muestreo utilizado fue no probabilístico por conveniencia.

Localización. Los ovinos Sudán Blanco se localizaron en los municipios de San Juan del Cesar, La Guajira (10049'46.0" Lat. Norte -72056'53.8" Long. Oeste) y Valledupar, Cesar (10025'36.6" Lat. Norte -73021'16.0" Long. Oeste), mientras que los Sudán Bayo se encontraron distribuidos en los municipios de Valledupar, Cesar (10007'37.3' Lat. Norte $-73039^{\prime} 45.4^{\prime \prime}$ Long. Oeste y $10^{\circ} 07^{\prime} 01.4^{\prime \prime}$ Lat. Norte - 73033'02.2" Long. Oeste), Montería y Ciénaga de Oro, Córdoba (8034'15.7" Lat. Norte - 75056'54.5" Long. Oeste y 8052'43.1" Lat. Norte - 75042'12.3" Long. Oeste). Los municipios pertenecientes a La Guajira y Cesar se consideran como Bosque Muy Seco Tropical y los municipios de Córdoba se catalogan como Bosque Seco Tropical (5).

Animales del estudio. El primer reporte en la literatura en el cual se describe a los OPC Sudán fue realizado por Bautista (2), quien los definió básicamente como ovejas de pelaje que va desde amarillo hasta blanco, perfil "rectilíneo semiconvexo", sin cuernos, orejas cortas y en forma horizontal, ojos de color café o amarillos, con presencia de mamellas ocasionalmente y de pezuñas de color claro o pigmentado.

Dentro del subgrupo Sudán se encuentran los Sudán Bayo (Figura 1) y Sudán Blanco (Figura 2), el cual a su vez pertenece al grupo denominado Ovinos de Pelo Criollos Colombianos (OPC), grupo en el que hay al menos otro subgrupo llamado Etiope, siendo estos OPC en general conocidos como Camuros, Ovejas Africanas, Pelonas, Criollas, entre otras diversas maneras acorde a la ubicación geográfica. A pesar de que los Sudán Bayo y Sudán Blanco pertenecen al mismo subgrupo y son similares entre sí, ambos son criados apartados y como poblaciones diferentes, siendo estos últimos marcadamente escasos con respecto a los primeros.

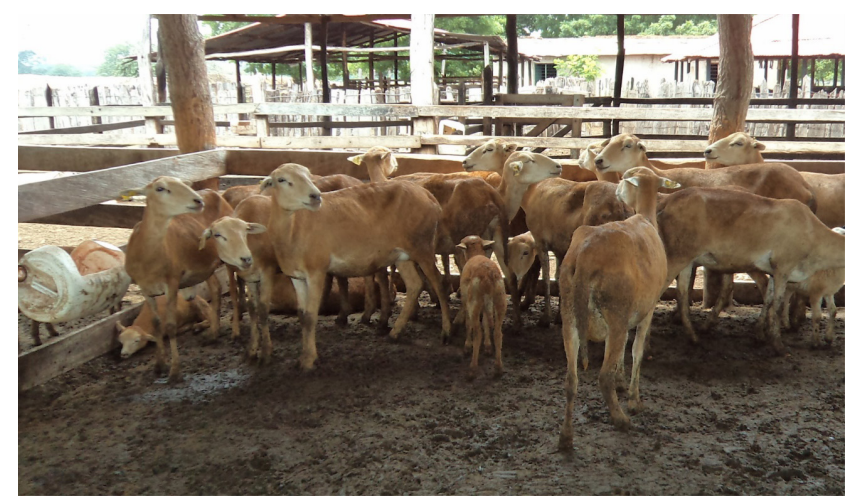

Figura 1. Ovinos Sudán Bayo.

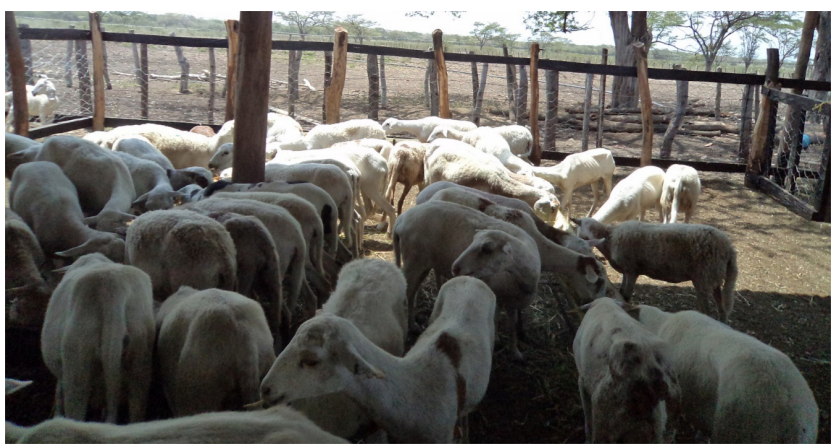

Figura 2. Ovejas Sudán Blanco.

Manejo animal y características evaluadas. Para realizar la caracterización morfológica de los ovinos, se seleccionaron ejemplares que concordaran con la descripción fenotípica del OPC Sudán encontrada en la literatura (2). El número de ejemplares evaluados fue de 115, siendo 87 hembras Sudán Bayo y 28 hembras Sudán Blanco, las cuales fueron mayores a un año. Se descartó cualquier animal que presentara anomalías anatómicas, que evidenciara cruzamiento con otra raza, enfermedad o que estuviera en estado de preñez.

La evaluación de las características morfológicas y fanerópticas fue hecha a través de la inspección visual, con apoyo de una cámara fotográfica y un goniómetro digital. Este último instrumento se utilizó con el fin de tener una mayor precisión valorando el carácter relativo a la grupa; esta información fue anotada en formatos de recolección elaborados por los autores. Por ser de naturaleza subjetiva la evaluación de estas características siempre hubo unificación de criterio entre los autores en cada calificación.

Para escoger las características fanerópticas y morfológicas cualitativas estudiadas, se tomaron como referencias los trabajos realizados por De la Barra et al (10), Peña et al (11), De la Rosa 
et al (12), Bravo y Sepúlveda (13), Hick (14) y Montes et al (15). De los primeros autores se tuvo en cuenta el color de la capa, de los segundos el color de la piel, de los terceros la dirección de las orejas y pigmentación de las mucosas, de los cuartos la pigmentación de la ubre, característica del vientre y perfil cefálico, del quinto pigmentación de las pezuñas y de los sextos la apariencia del cuello, inclinación de la grupa y profundidad de la ubre; la presencia de mamellas y el largo del cuello se incluyeron por iniciativa de los autores, con el fin de dar una descripción más detallada de los OPC Sudán.

\section{Características fanerópticas:}

Color de la capa (CCa). Las ovejas Sudán Bayo tuvieron tres tonalidades que fueron bayo claro, bayo amarillo y bayo encerado, mientras que para las Sudán Blanco se consideraron dos capas, blanco y overo castaño. Todas las características que se describen a continuación, se evaluaron bajo los mismos criterios tanto en ovejas Sudán Bayo como en Sudán Blanco.

Color de la piel (CP). Esta característica se evaluó en el dorso y sobre la región costal, teniéndose en cuenta cuatro colores que van desde el sonrosado, hasta el pigmentado total, pasando por el blanco cera y el pigmentado parcial.

Pigmentación de las mucosas (PMu). Se observó la pigmentación teniendo en cuenta el morro, los labios y la lengua, clasificándose como rosada, negra y negra-rosada (Figura 3 ).

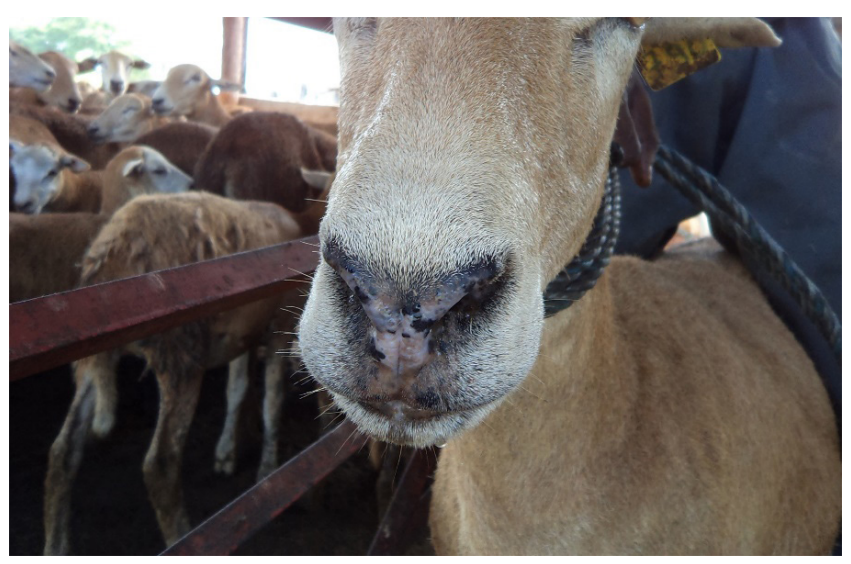

Figura 3. Morro y labios de pigmentación negra-rosada.

Presencia de mamellas (PM). Para esta característica se analizó si había presencia o ausencia de mamellas, también llamadas marmellas, zarcillos o pendientes. Las mamellas son apéndices cartilaginosos que se ubican en la parte superior del borde traqueal del cuello.
Pigmentación de pezuñas (PP). Con base en la pigmentación se consideraron tres tipos: veteadas, claras y oscuras, siendo la primera una pigmentación parcial.

Pigmentación de la ubre (PU). Para la evaluación de dicha pigmentación se utilizaron los criterios de ubre no pigmentada, pigmentada y parcialmente pigmentada (Figura 4).

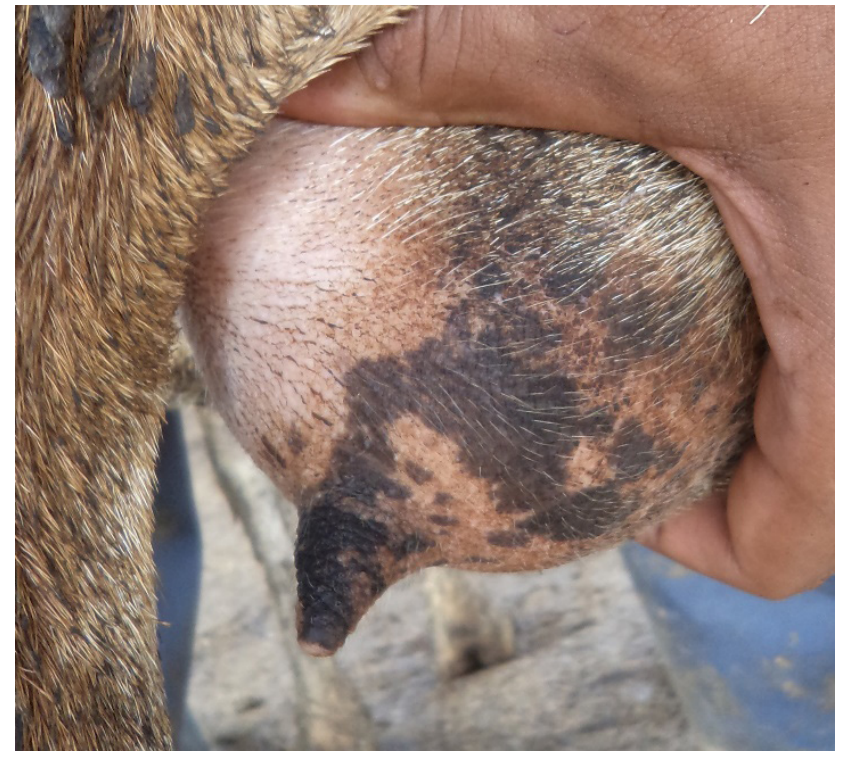

Figura 4. Ubre parcialmente pigmentada.

\section{Características morfológicas cualitativas:}

Dirección de las orejas (DO). Se valoró si estas estaban dirigidas horizontalmente, eran erguidas o por el contrario caídas (Figura 5).

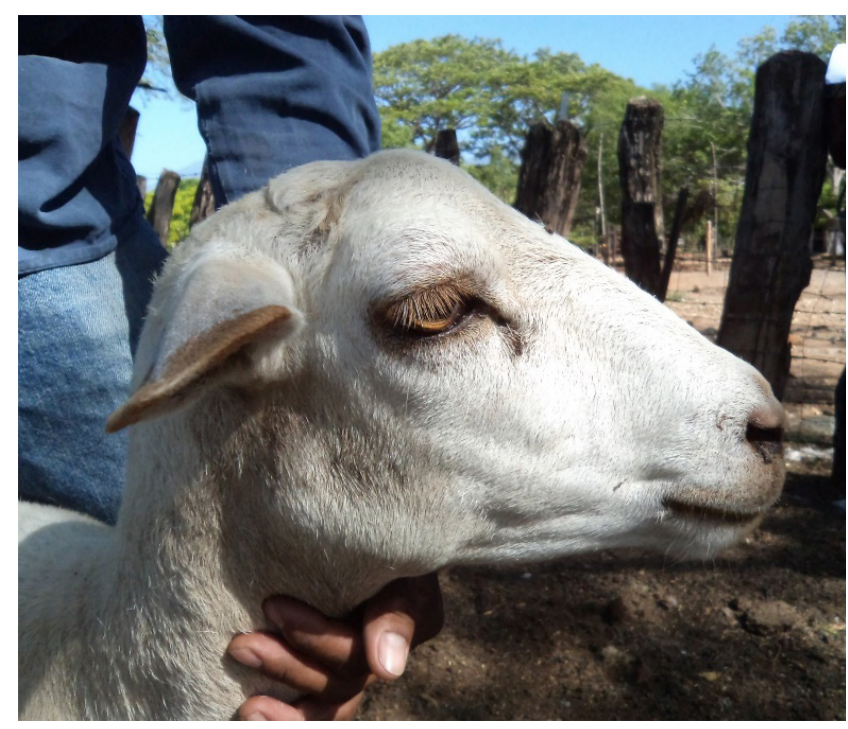

Figura 5. Ejemplar Sudán Blanco de orejas horizontales y perfil recto. 
Perfil cefálico (PCe). Con base al aloidismo, se contemplaron los perfiles naso-frontales cóncavo, recto y convexo, teniéndose en cuenta además los puntos intermedios entre estos, es decir, subcóncavo y subconvexo (Figura 5).

Longitud del cuello (LCu). Se evaluó de acuerdo a la proporción que presentaron las ejemplares entre el largo del cuello y el tronco, calificándose como largo, mediano o corto (Figura 6).

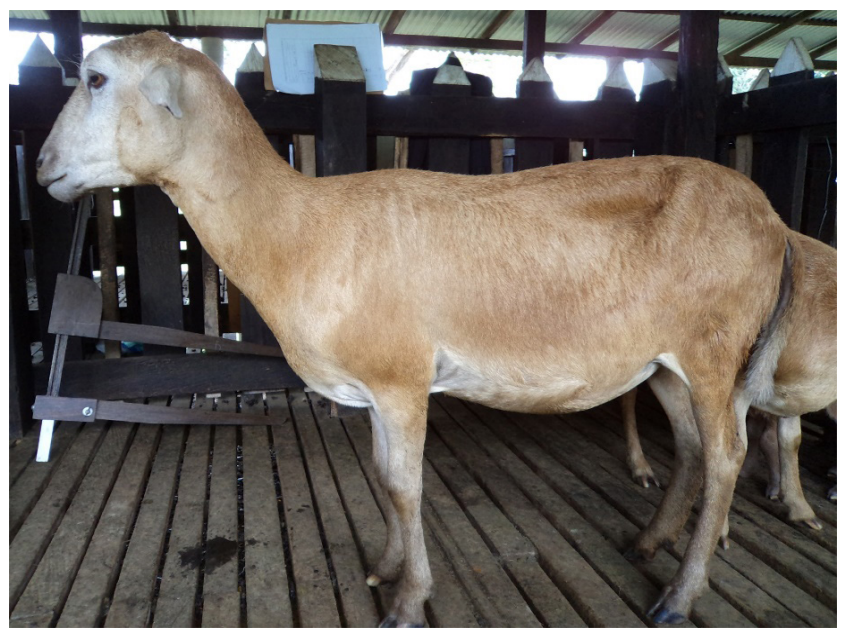

Figura 6. Vista desde el costado para la evaluación del cuello y del vientre.

Apariencia del cuello (ACu). Para evaluar esta característica se consideró su proporcionalidad con respecto al cuerpo, y fue catalogado como poco musculado, moderadamente musculado o muy musculado (Figura 6).

Característica del vientre (CVe). De acuerdo con la curvatura ventral, se categorizó a las ovejas como vientres de galgo, vientres recogidos o ventrudas (Figura 6 ).

Inclinación de la grupa (IG). Cuando el ángulo era igual o inferior a $20^{\circ}$ la grupa se catalogaba como recta, cuando la inclinación se presentaba mayor a $20^{\circ}$ y menor a $25^{\circ}$ la clasificación era levemente recta, si presentaba una grupa que oscilaba entre los $25^{\circ}$ y $35^{\circ}$ se denominaba inclinada, si la angulación era mayor a $35^{\circ}$ y menor a $40^{\circ}$ se consideraba como levemente derribada y si iba desde los $40^{\circ}$ hacia delante se clasificaba como grupa derribada.

Profundidad de la ubre (PFu). Es la distancia entre la inserción posterior y la base de la ubre, cuya calificación se hace en relación con la alzada de la ejemplar, teniendo como referencia el corvejón. Para evaluar este carácter se catalogaron las ubres como muy profundas, medianamente profundas y poco profundas, siendo la primera calificación dada con una puntuación de nueve a siete, la segunda con una valoración de seis a cuatro y la tercera cuando la calificación era de tres a uno; a medida que decrecían los números era menor la profundidad (16).

Análisis de los resultados. La información recolectada fue transcrita a Microsoft Excel ${ }^{\circledR}$ 2013 y posteriormente depurada, para dar paso a la estimación de las frecuencias relativas y absolutas, a través del programa estadístico InfoStat ${ }^{\circledR}$ versión 2016I (17).

\section{RESULTADOS}

Características fanerópticas. Los resultados de las características fanerópticas se presentan en la tabla 1. Como primer carácter evaluado está el CCa, donde más del $90 \%$ de ovejas Sudán Bayo presentaron un color bayo amarillo, estando el porcentaje restante distribuido en CCa bayo claro $(8.05 \%)$ y bayo encerado $(1.15 \%)$. Más de la mitad de las Sudán Blanco presentaron un CCa overo castaño (60.71\%), o sea, blanco con manchas castaño, pero siempre predominando el blanco respecto a las manchas de color castaño, manchas que incluso generalmente se presentaban pequeñas y/o sólo en la cabeza o en una delimitada región del cuerpo. El porcentaje restante de las Sudán Blanco se caracterizó por tener una capa completamente blanca.

En el caso del $\mathrm{CP}$, en todas las ovejas Sudán el color fue blanco cera, a excepción de una ejemplar Sudán Bayo, la cual presentó un CP sonrosado. En cuanto a la PMu, se observó que en las Sudán Bayo el $100 \%$ presentaron mucosas negra-rosadas, predominando el negro sobre el rosado o viceversa. Por su parte, en las hembras Sudán Blanco el $39.29 \%$ tuvo mucosas rosadas y el resto negra-rosadas.

La ausencia de mamellas fue una característica mostrada por gran parte de las ovejas Sudán Bayo $(85.06 \%)$ y Blanco $(75 \%)$, mientras que los porcentajes restantes de las ejemplares, que enmarcan una considerable cifra de la población, sí presentaron mamellas.

En lo concerniente a la PP, se obtuvo que las Sudán Bayo ostentaron pezuñas veteadas casi en la totalidad de las ovejas, a excepción de un individuo cuyas pezuñas fueron claras, o sea, sin pigmentación. En las Sudán Blanco se presentó un comportamiento distinto para esta característica, puesto que, aunque el mayor porcentaje de la población estudiada presentó pezuñas veteadas, un $42.86 \%$ las presentaban claras. 
Flórez et al - Caracterización morfológica de ovejas Sudán OPC

Tabla 1. Frecuencias absolutas y relativas de las características fanerópticas de hembras Sudán Bayo y Sudán Blanco.

\begin{tabular}{|c|c|c|c|c|c|}
\hline \multirow{2}{*}{\multicolumn{2}{|c|}{ Características fanerópticas }} & \multicolumn{2}{|c|}{ SudánBayo } & \multicolumn{2}{|c|}{ SudánBlanco } \\
\hline & & FA* & $\mathrm{FR}(\%) * *$ & FA & FR (\%) \\
\hline \multirow{3}{*}{ Color de la capa $(\mathrm{CCa}) * * *$} & 1 & 7 & 8.05 & 11 & 39.29 \\
\hline & 2 & 79 & 90.8 & 17 & 60.71 \\
\hline & 3 & 1 & 1.15 & - & - \\
\hline \multirow{4}{*}{ Color de la piel (CP) } & Sonrosado & 1 & 1.15 & 0 & 0 \\
\hline & Blanco cera & 86 & 98.85 & 28 & 100 \\
\hline & Pigmentado parcialmente & 0 & 0 & 0 & 0 \\
\hline & Pigmentado totalmente & 0 & 0 & 0 & 0 \\
\hline \multirow{3}{*}{ Pigmentación de las mucosas (PMu) } & Rosadas & 0 & 0 & 11 & 39.29 \\
\hline & Negra-rosadas & 87 & 100 & 17 & 60.71 \\
\hline & Negras & 0 & 0 & 0 & 0 \\
\hline \multirow{3}{*}{ Presencia de mamellas (PM) } & $\mathrm{Si}$ & 13 & 14.94 & 7 & 25 \\
\hline & No & 74 & 85.06 & 21 & 75 \\
\hline & Claras & 1 & 1.15 & 12 & 42.86 \\
\hline \multirow[t]{3}{*}{ Pigmentación de pezuñas (PP) } & Veteadas & 86 & 98.85 & 16 & 57.14 \\
\hline & Oscuras & 0 & 0 & 0 & 0 \\
\hline & $\mathrm{Si}$ & 2 & 2.3 & 0 & 0 \\
\hline \multirow[t]{2}{*}{ Pigmentación de la ubre (PU) } & Parcial & 70 & 80.46 & 14 & 50 \\
\hline & No & 15 & 17.24 & 14 & 50 \\
\hline
\end{tabular}

* FA: frecuencia absoluta. ** FR: frecuencia relativa en porcentaje.

*** Sudán Bayo: 1. Bayo claro, 2. Bayo amarillo, 3. Bayo encerado; Sudán Blanco: 1. Blanco, 2. Overo castaño.

Como última característica faneróptica se tuvo la PU, la cual se caracterizó por ser de pigmentación parcial en el $80.46 \%$ de las Sudán Bayo y el $50 \%$ de las Sudán Blanco, teniendo el otro $50 \%$ de ovejas Sudán Blanco ubres despigmentadas, al igual que un $17.24 \%$ de las Sudán Bayo; sólo dos ejemplares Sudán Bayo fueron de ubres pigmentadas.

Características morfológicas cualitativas. Los resultados de estas siete características se presentan en la tabla 2.

Tabla 2. Frecuencias absolutas y relativas de las características morfológicas cualitativas de hembras Sudán Bayo y Sudán Blanco.

\begin{tabular}{|c|c|c|c|c|c|c|}
\hline \multirow{2}{*}{\multicolumn{3}{|c|}{ Características morfológicas cualitativas }} & \multicolumn{2}{|c|}{ Sudán Bayo } & \multicolumn{2}{|c|}{ Sudán Blanco } \\
\hline & & & FA* & FR (\%)** & FA & FR (\%) \\
\hline & \multicolumn{2}{|l|}{ Horizontales } & 87 & 100 & 28 & 100 \\
\hline \multirow[t]{4}{*}{ Dirección de las orejas (DO) } & \multicolumn{2}{|l|}{ Erguidas } & 0 & 0 & 0 & 0 \\
\hline & \multicolumn{2}{|l|}{ Caídas } & 0 & 0 & 0 & 0 \\
\hline & \multicolumn{2}{|l|}{ Cóncavo } & 0 & 0 & 0 & 0 \\
\hline & \multicolumn{2}{|l|}{ Subcóncavo } & 0 & 0 & 0 & 0 \\
\hline \multirow[t]{4}{*}{ Perfil cefálico (PCe) } & \multicolumn{2}{|l|}{ Recto } & 13 & 14.94 & 5 & 17.86 \\
\hline & \multicolumn{2}{|l|}{ Subconvexo } & 74 & 85.06 & 23 & 82.14 \\
\hline & \multicolumn{2}{|l|}{ Convexo } & 0 & 0 & 0 & 0 \\
\hline & \multicolumn{2}{|l|}{ Largo } & 11 & 12.64 & 8 & 28.57 \\
\hline \multirow[t]{3}{*}{ Longitud del cuello ( $\mathrm{LCu}$ ) } & \multicolumn{2}{|l|}{ Mediano } & 73 & 83.91 & 13 & 46.43 \\
\hline & \multicolumn{2}{|c|}{ Corto } & 3 & 3.45 & 7 & 25 \\
\hline & \multicolumn{2}{|c|}{ Poco musculada } & 55 & 63.22 & 28 & 100 \\
\hline \multirow[t]{3}{*}{ Apariencia del cuello (ACu) } & \multicolumn{2}{|c|}{ Moderadamente musculada } & 32 & 36.78 & 0 & 0 \\
\hline & \multicolumn{2}{|c|}{ Muy musculada } & 0 & 0 & 0 & 0 \\
\hline & \multicolumn{2}{|c|}{ Vientre de galgo } & 0 & 0 & 0 & 0 \\
\hline \multirow[t]{4}{*}{ Característica del vientre (CVe) } & \multicolumn{2}{|c|}{ Vientre recogido } & 22 & 25.29 & 16 & 57.14 \\
\hline & \multicolumn{2}{|c|}{ Ventrudo } & 65 & 74.71 & 12 & 42.86 \\
\hline & \multicolumn{2}{|c|}{ Grupa recta } & 0 & 0 & 0 & 0 \\
\hline & \multicolumn{2}{|c|}{ Grupa levemente recta } & 15 & 17.24 & 8 & 28.57 \\
\hline \multirow[t]{6}{*}{ Inclinación de la grupa (IG) } & \multicolumn{2}{|c|}{ Grupa inclinada } & 66 & 75.86 & 20 & 71.43 \\
\hline & \multicolumn{2}{|c|}{ Grupa levemente derribada } & 6 & 6.9 & 0 & 0 \\
\hline & Grupa derriba & & 0 & 0 & 0 & 0 \\
\hline & & 1 & 15 & 17.24 & 10 & 35.71 \\
\hline & Ubres poco profundas & 2 & 38 & 43.68 & 5 & 17.86 \\
\hline & & 3 & 24 & 27.59 & 10 & 35.71 \\
\hline Profundidad de la ubre (PFu) & & 4 & 6 & 6.9 & 0 & 0 \\
\hline & Ubres medianamente & 5 & 4 & 4.6 & 3 & 10.72 \\
\hline & prorundas & 6 & 0 & 0 & 0 & 0 \\
\hline & Ubres muy profundas & $7,8,9$ & 0 & 0 & 0 & 0 \\
\hline
\end{tabular}

* FA: frecuencia absoluta. ** FR: frecuencia relativa en porcentaje. 
La DO se caracterizó por ser en el $100 \%$ de las OPC Sudán de forma horizontal. En relación al PCe, más del $80 \%$ de las Sudán presentaron un perfil subconvexo, estando el porcentaje restante enmarcado en la categoría de perfil recto, más específicamente el $14.94 \%$ de las Sudán Bayo y el $17.86 \%$ de las Blanco.

En relación a la $\mathrm{LCu}$ de las Sudán Bayo, el mayor porcentaje de la población (83.91\%) presentó una LCu mediana, siendo el menor número el de las ejemplares de LCu corto $(3.45 \%)$ y el porcentaje restante fue asignado a los individuos de cuello largo (12.64\%). En las Sudán Blanco más de la mitad de las hembras se encontró distribuida en longitudes para el cuello catalogadas como largas $(28.57 \%)$ y cortas $(25 \%)$, mientras que el porcentaje restante de las ovejas se enmarcaron entre lo considerado como de una LCu mediana. Todas las Sudán Blanco evaluadas y el $63.22 \%$ de las Sudán Bayo tuvieron un cuello de apariencia poco musculada, mientras que el porcentaje restante de estas últimas se consideraron como de cuellos de apariencia moderadamente musculada.

En la CVe se obtuvo que en las Sudán Bayo el $74.71 \%$ de las ovejas fueron ventrudas y las restantes de vientres recogidos, ocurriendo lo contrario para las Sudán Blanco, donde más de la mitad (57.41\%) presentaron vientres recogidos y en menor porcentaje (42.59\%) fueron ventrudas. Respecto a la IG, gran parte de hembras Sudán presentaron una grupa que se caracterizaba por ser inclinada $(>70 \%)$, siendo el porcentaje restante en Sudán Blanco con IG levemente recta (28.57\%). Mientras que en Sudán Bayo dicho porcentaje restante se distribuyó minoritariamente en grupas levemente derribadas $(6.9 \%)$ y en levemente rectas $(17.24 \%)$.

Como último carácter se tuvo a la PFu, la cual permitió catalogar a la mayor parte de las Sudán como de ubres poco profundas, más específicamente el $88.51 \%$ de las ovejas Sudán Bayo y el $89.28 \%$ de las Sudán Blanco; las hembras restantes se clasificaron como de ubres medianamente profundas.

\section{DISCUSIÓN}

Características fanerópticas. Las Sudán Bayo independiente de la tonalidad de bayo de la capa, presentaron degradación de esta a nivel del hocico, los ojos, las orejas, la bragada, la región interaxilar, el vientre y algunas veces y menos notoriamente en las extremidades; la intensidad de la degradación varió entre los individuos. Estas características de degradación también habían sido expuestas por Bautista (2) en estas OPC, quien afirmó que el bayo tendía a degradarse en la porción terminal de los ejemplares.

Aunque en la literatura no se menciona un CCa blanco con pequeñas y pocas manchas cafés (overo castaño) para los Sudán, sino un CCa que es casi blanco, los autores decidieron catalogar a los individuos de CCa overo castaño como Sudán Blanco, debido a que sus ascendientes y descendientes eran de un CCa como el de ellos o completamente blanco, siendo una prioridad para los propietarios que no hubiese introgresión de individuos de otra raza en estos rebaños. Además, los criadores de Sudán Blanco no discriminaban sobre si el CCa era blanco u overo castaño. Por lo tanto, los autores consideraron que lo indicado fue que se incluyera en los Sudán Blanco el CCa overo castaño, lo cual es un reflejo de que la faneróptica de los OPC Sudán en la actualidad probablemente es diferente a lo hallado hace cuatro décadas por Bautista (2), quien fue el primero en describir a los OPC Sudán y el único que lo ha hecho hasta el momento, desde la faneróptica y la morfología cualitativa diferenciándolos de los otros "tipos" de OPC.

En el estudio hecho por Montes et al (15), quienes evaluaron el CCa de OPC en Sucre, se encontró que el 24\% fueron amarillos (bayos), el $45 \%$ cafés (similar al rojo), el $12 \%$ blancos, el $7 \%$ negros y el porcentaje restante correspondía a la combinación de varios colores. No obstante, ovinos con un CCa que se salga del rango que hay entre las tonalidades que van desde el bayo hasta el blanco, no son considerados como OPC Sudán (2), y en ocasiones se encuentran en los mismos hatos que estos, ya que suelen ser cruces de OPC con razas foráneas, haciendo la salvedad de que ello no aplica cuando los OPC son de un CCa de tonalidades rojizas, pues este es el principal rasgo distintivo de los OPC Etiope (2).

En algunas de las OPC Sudán Bayo y Sudán Blanco estudiadas, se evidenció la presencia de rudimentos de lana en la región dorsal y lumbosacra, lo que se considera como una respuesta fenotípica al ambiente, especialmente a la exposición prolongada a radiaciones solares, la cual probablemente se hace posible por hibridaciones en el pasado de los OPC con individuos lanados. Esto se sustenta en que 
Vivas (4) encontró que no es mucha la distancia genética de los OPC de Córdoba, con las razas ovinas criollas lanadas de los departamentos de Nariño y Boyacá.

Contrario a lo encontrado en este estudio para la PMu, donde las Sudán fueron de mucosas negra-rosadas o rosadas, Montes et al (15) reportaron que la mayoría $(82 \%)$ de OPC que ellos evaluaron presentaban PMu negras. Por su parte, Bravo y Sepúlveda (13) hallaron en Ovejas Criollas Araucanas, que el $50 \%$ poseían mucosas negras, el $46.81 \%$ oscurecidas y el porcentaje restante rosadas.

En el contexto productivo existen hipótesis sobre que la PM otorga mayor pureza racial en las OPC, o que este es un factor que incluye o excluye en cuanto al hecho de pertenecer a esta población. Sin embargo, a través de los resultados de esta investigación se puede apreciar que es normal la presencia o ausencia de mamellas, por lo que a esta característica no se le deben atribuir méritos. Además, no existen investigaciones en las que de manera certera las mamellas se relacionen con aspectos reproductivos, por lo tanto, se sugiere que sean consideradas como una característica neutra en la selección.

Aunque en las Sudán Bayo y Blanco no se encontraron ejemplares de pezuñas pigmentadas, este tipo de pezuñas fueron reportadas en OPC por Montes et al (15) en el $28.6 \%$ de la población, mientras que Bautista (2) plantea que en las Sudán es normal la existencia de pezuñas claras, tal como se halló en el actual estudio.

Las ovejas Sudán en su mayoría presentaron ubres parcialmente pigmentadas, predominando usualmente la despigmentación con respecto a las áreas pigmentadas, no obstante, Montes et al (15) no encontraron hembras OPC con esta característica, siendo el $96.7 \%$ de las ejemplares de dicho estudio de ubres despigmentadas.

Características morfológicas cualitativas. Al igual que en el presente trabajo, Bautista (2) encontró que las OPC Sudán poseían orejas direccionadas horizontalmente, resultado similar al reportado en OPC del departamento de Sucre (15), donde el 99\% de las ovejas presentaron una DO horizontal. En hembras de la raza Criolla Araucana, se halló que el $97.87 \%$ poseían orejas horizontales y el $2.13 \%$ orejas caídas (13).
El PCe hallado en las hembras Sudán Bayo y Blanco discrepa de manera marcada con el de las OPC de Sucre (15), las cuales fueron catalogadas de PCe generalmente recto, lo cual concuerda con menos del $15 \%$ en hembras Sudán Bayo y $18 \%$ en Sudán blanco. Estas diferencias posiblemente se deben a que Montes et al (15) no utilizaron en su evaluación las clasificaciones intermedias de subcóncavo y subconvexo, por lo que esta última al tener una leve tendencia a la rectitud, se pudo tomar como PCe recto. Además, otro factor que probablemente influyó fue el componente genético, ya que, aunque Montes et al (15) realizaron su trabajo en OPC, sólo el $36 \%$ de los individuos presentaron un CCa concordante con el clásico de las OPC Sudán.

Respecto a la $\mathrm{LCu}$, lo reportado por Bautista (2) es contrario a lo encontrado en las Sudán, puesto que este autor describe a los OPC Sudán como de cuellos largos, cuando en la actual investigación las ejemplares con una LCu catalogada como larga no superó el $13 \%$ en las Sudan Bayo y el $29 \%$ en Sudan Blanco, ya que la mayoría de las ovejas Sudán fueron de una LCu mediana. Lo hallado en las Sudán para la CVe es concordante con lo expuesto por Bautista (2) para esta característica, lo que confirma que estas OPC suelen ser ventrudas.

Grupas inclinadas, al igual que las grupas de la mayor parte de las Sudán del actual trabajo, se reportaron en un $97.4 \%$ de hembras OPC de Sucre (15); sólo el $2.6 \%$ de las ovejas del previo estudio presentaron una IG recta. Las grupas inclinadas del anterior trabajo posiblemente fueron más frecuentes que las de las Sudán, debido a que el sistema de clasificación usado en esta investigación fue más específico, sin embargo, ambos eran similares.

Por último, Montes et al (15) hallaron en ovejas OPC para la PFu, que estas se caracterizan por ser de ubres poco profundas (71.1\%). Ubres de poca profundidad también fueron encontradas en las OPC Sudán del presente estudio. La reducida PFu fue más marcada en las Sudán Bayo y Blanco que en las OPC del departamento de Sucre (15).

En Ovejas Criollas Araucanas (13) no se evaluó la PFu propiamente dicha. Sin embargo, se valoró el tamaño, catalogándose así las ubres de estos ovinos como pequeñas para el $97.87 \%$ de los casos, lo que permite inferir que las Criollas Araucanas, al igual que la mayor parte de las Sudán, eran de ubres poco profundas, resultados que eran de esperar, pues ambas razas están orientadas hacia la producción de carne. 
Con base a las variables fanerópticas y morfológicas cualitativas evaluadas, se concluye que las hembras OPC Sudán se caracterizan por lo expuesto a continuación.

Por estar revestidas de pelos cortos y lisos, cuya longitud disminuye en la cabeza, la región ventral y a lo largo de las extremidades, especialmente hacia la parte inferior de estas; poseer ocasionalmente rudimentos de lana; ser acorneas. Por siempre tener un CCa bayo las Sudán Bayo, mientras que en las Sudán Blanco aparte de encontrarse ejemplares de capa completamente blanca, es común la presencia de individuos de capa overo castaño.

Suelen poseer un color de piel blanco ceroso, presentan mucosas de pigmentación negrarosada en la mayoría de los casos. Tienen las orejas siempre orientadas de manera horizontal y con desprovisión de pelo en el pabellón auricular interno. Presentan PCe subconvexos, aunque suelan ser de frente recta, siendo además normal, pero menos frecuentes perfiles cefálicos rectos en su totalidad, los cuales favorecen que sobresalgan las órbitas y se forme una depresión detrás de cada arco orbitario; se caracterizan por ser de ojos color ámbar.

Generalmente tienen cuellos de forma cónica, de longitud mediana y de apariencia poco musculada, aunque también hay ejemplares de cuellos moderadamente musculados; una considerable proporción de las Sudán presentan mamellas; la forma del tronco suele ser cilíndrica. Las Sudán Bayo destacan por ser ventrudas y las Sudán Blanco por ser vientre recogido, sin embargo, ambas características suelen estar presentes en ambas poblaciones. Presentan normalmente grupas inclinadas; poseen pezuñas que en gran parte de los individuos son veteadas; ostentan ubres que generalmente son de poca profundidad y que suelen ser parcialmente pigmentadas.

Aunque hubiese sido ideal una muestra poblacional de mayor tamaño, se sugiere que los resultados de esta primera etapa de caracterización morfológica y faneróptica de las Sudán, son de elevada importancia, puesto que proporcionan información detallada y sobre el estado actual de los rasgos exteriores de este grupo de OPC en específico, datos que en caso de que se encontraran para esta población en particular eran básicos y además, estos presentaron diferencias con los estudios que no discriminaban sobre la clase de OPC estudiada, Sudán o Etiope.

Por lo tanto, estos resultados han permitido caracterizar a las Sudán y serán de utilidad para consensar sobre la adecuada clasificación racial de los OPC, para subsiguientemente definir un estándar racial de los Sudán, de tal modo que se establezcan desde los parámetros discriminatorios que definen la pertenencia o no a esta población, hasta los que marquen las pautas hacia dónde se debe dirigir la selección, desde la perspectiva morfológica cualitativa y faneróptica; pudiendo repercutir todo ello finalmente, en el máximo aprovechamiento por parte de los productores de este recurso zoogenético, lo que a su vez impactaría positivamente en la conservación y protección de Ios OPC Sudán, quienes han demostrado su adaptabilidad al trópico bajo colombiano.

\section{REFERENCIAS}

1. Delgado JV, Perez Grovas R, Camacho ME, Fresno M, Barba C. The Wool-less Canary sheep and their relationship with the present breeds in America. Anim Genet Resour. 2000; 28:27-34. https://doi.org/10.1017/ $\underline{\mathrm{S} 1014233900001334}$

2. Bautista Otero R. Apuntes Sobre Ovino Africano en Colombia. Carta Agraria (ed.) Carta Agraria No 277. Colombia: Caja de Crédito Agrario; 1980.
3. Aguilar Martínez CU, Berruecos Villalobos JM, Espinoza Gutiérrez B, Segura Correa JC, Valencia Méndez J, Roldán Roldán A. Origen, historia y situación actual de la oveja Pelibuey en México. Trop Subtrop Agroecosyst 2017; 20:429-439. http:// www.revista.ccba.uady.mx/ojs/index.php/ TSA/article/view/2348/1085

4. Vivas Ascue NJ. Diversidad genética de ovinos criollos Colombianos. [Tesis de Maestría]. Palmira, Colombia: Universidad Nacional de Colombia; 2013. http://bdigital. unal.edu.co/12683/ 
5. Flórez Murillo JM, Hernández Pérez $M$ de J, Bustamante Yánez M de J, Vergara Garay OD. Caracterización morfoestructural de tres poblaciones de Ovino de Pelo Criollo Colombiano "OPC". Arch Zootec 2018; 67(259):340-348. https://doi. org/10.21071/az.v67i259.3789

6. Simanca J, Vergara O, Bustamante M. Description of growth in sheep creole (Ovis aries) in two populations from Córdoba, Colombia. Rev MVZ Córdoba 2017; 22(3):6310-6319. http://dx.doi. org/10.21897/rmvz.1135

7. Acero Plazas VM. El bienestar animal en sistemas productivos de ovinos-caprinos en Colombia. Spei Domus 2014; 10(21):57-62. https://doi.org/10.16925/sp.v10i21.918

8. Parra RI, Magaña MA, Duarte JH, Téllez G. Caracterización técnica y rentabilidad de granjas ovinas con visión empresarial del departamento del Tolima. Rev Colombiana Cienc Anim - RECIA. 2014; 7(1):6472. http://revistas.ut.edu.co/index.php/ ciencianimal/article/view/543

9. Sierra I. Importancia de la morfología y su valoración en los animales domésticos. En: Sañudo C dir. Valoración morfológica de los animales domésticos. Madrid: Ministerio de Medio Ambiente y Medio Rural y Marino; 2009. https://www.mapa.gob.es/ es/ganaderia/temas/zootecnia/LIBRO\%20 valoracion $\% 20$ morfologica $\% 20$ SEZ tcm 30 119157.pdf

10. De la Barra R, Martínez ME, Calderón C. Phenotypic features and fleece quantitative traits in Chilota sheep breed. J Livestock Sci 2014; 5:28-34. http://livestockscience.in/ wp-content/uploads/De-la-Barra-2014.pdf

11. Peña S, López G, Martínez R, Abbiati N, Género E, Guarófalo M. Relevamiento morfológico de ovinos criollos en cuatro regiones de la Argentina. Informe preliminar. AICA 2012; 2:61-66. https://aicarevista.jimdo.com/ n\%C3\%BAmeros/vol\%C3\%BAmen-2-2012/
12. De la Rosa SA, Revidatti MA, Tejerina ER, Orga A, Cappello JS, Petrina JF. Estudio para la caracterización de la oveja criolla en la región semiárida de Formosa, Argentina. AICA 2012; 2(1):87-94. http://www.uco.es/ conbiand/aica/templatemo 110 lin photo/ articulos/2012/Trabajo041 AICA2012.pdf

13. Bravo S, Sepúlveda N. Índices zoométricos en ovejas criollas Araucanas. Int J Morphol. 2010; 28(2):489-495. http://dx.doi. org/10.4067/S0717-95022010000200025

14. Hick MV. Caracterización etnozootécnica de poblaciones primarias (criollas) de ovinos, caprinos y camélidos domésticos productores de fibra. [Tesis Doctoral]. Córdoba, Argentina: Universidad Católica de Córdoba; 2015. http://pa.bibdigital.uccor. edu.ar/137/

15. Montes Vergara D, Moreno J, HurtadoLugo N, Ramírez R, Celis A y Garay G. Caracterización faneróptica y morfológica de la hembra ovina de pelo criollo (Camura) colombiana, en la Sub Región Sabanas y Golfo de Morrosquillo departamento de Sucre. Rev Colombiana Cienc Anim. 2013; 5(1): 104-115. https://doi.org/10.24188/ recia.v5.n1.2013.475

16. Castañares N. Recría de corderas de raza Assaf Española: efecto de la alimentación entre el nacimiento y los cinco meses de edad sobre el crecimiento de los animales, el desarrollo de la glándula mamaria y la producción de leche en la primera lactación. [Tesis Doctoral]. León, España: Universidad de León; 2008. https://digital.csic.es/ handle/10261/5237

17. Di Rienzo JA, Casanoves F, Balzarini MG, Gonzalez L, Tablada M, Robledo YC. InfoStat. Versión 2016. Argentina: Universidad Nacional de Córdoba; 2016. https://www. infostat.com.ar/ 